\title{
Literatura francesa de viajes y códigos QR. Espacios culturales, espacios interculturales
}

\author{
French travel literature and QR codes. \\ Cultural spaces, intercultural spaces
}

\author{
CARlota Vicens-Pujol \\ Universidad de las islas Baleares \\ cvicens@uib.es
}

\begin{abstract}
Résumé
Le présent travail entend analyser la citation littéraire insérée dans ce que nous appellerons les Guides de voyage QR. Plus précisément, nous verrons quels textes, de quels écrivains français (Camus ou Bernanos parmi d'autres) ont été choisis pour faire partie de l'application "WoW Mallorca Literaria", lancée en 2016 par le Consell Insular de Mallorca avec la prétention de faire connaître le patrimoine littéraire de l'île. Après une analyse des particularités de ce moderne guide de voyage littéraire, nous essaierons de répondre à quelques questions concernant l'altérité et l'interculturalité: Quelle image de l'autre et de soi-même ces citations offrent-elles? Est-ce qu'elles contribuent à créer une relation nouvelle de l'homme avec l'espace insulaire? Sommes-nous dans le domaine du culturel, de l'interculturel, à la frontière des deux? Diverses études sur l'interculturalité et l'intertextualité, ainsi que sur les affordances langagières constitueront la base de notre travail.
\end{abstract}

Mots-clés

littérature de voyage, interculturalité, intertextualité, WoW Mallorca Literaria.

\begin{abstract}
The present work analyzes the literary quotations inserted in what we call the QR Travel Guides. More precisely, we will see which texts of French writers (Camus or Bernanos, among others) were chosen to be part of the application "WoW Mallorca Literaria", launched in 2016 by the Consell Insular de Mallorca with the aim of promoting the island's literary heritage. After an analysis of the peculiarities of this modern literary travel guides, we will try to answer some questions concerning otherness and interculturality: What image of the "other" and of "oneself" do these quotes offer? Do they help to create a new human relationship with the island space? Are we in the field of culture, of interculturality, or are we on the border between both fields? Several studies on interculturality and intertextuality, as well as on language affordances, will form the basis of our paper.
\end{abstract}

\section{Key- words}

travel literature, interculturality, intertextuality, WoW Mallorca Literaria. 


\section{Introducción}

La culture, c'est la mémoire du peuple, la conscience collective de la continuité historique, le mode de penser et de vivre. Les livres et les tableaux ne sont que le miroir où cette culture profonde se reflète, se concentre, se conserve. (Milan Kundera')

Pertenece a Juan Ramón Jiménez la sentencia de que "en edición diferente los libros dicen cosa distinta" (Trapiello, 2021: 207). Muy lejos estaba entonces el poeta de imaginar que su Platero y yo había de entrar un día en el abismo de lo digital; que para leer sus poemas no sería necesario girar página alguna, sino arrastrar el dedo por una tableta o un smartphone; que la tipografía se acomodaría en nanosegundos a la agudeza visual del lector, cuando no a su simple capricho; que las páginas de sus libros, en fin, podrían estirarse a la manera de un acordeón, quedando la práctica totalidad de ellas en un inmerecido fuera de campo. Lícito es preguntarse si en una hipotética biblioteca post-borgiana, todos los libros estarían fuera de campo, pues metidos en un código QR, este módulo de almacenamiento de información en blanco y negro que la actual pandemia ha convertido en algo familiar. Para acabarnos de confundir, el escritor visionario que fue Borges incluyó su "Biblioteca de Babel" (1941) en los relatos de El jardín de senderos que se bifurcan. No sabemos si imaginó que serían tantas las bifurcaciones posibles...

No es nuestra intención hablar de Juan Ramón Jiménez ni de Jorge Luis Borges, pero sí de códigos QR en tanto que soportes materiales del discurso, lo que lleva a pensar en un "fuera de campo" discursivo, en un vacío que parece ser portador de significado. Partiremos para ello de un análisis de la aplicación "WoW Mallorca Literaria", destinada a un turismo literario, desde tres niveles, siendo el primero una aproximación a la misma en tanto que catalizadora del patrimonio literario insular y poniendo el acento en sus peculiaridades. En un segundo momento analizaremos la presencia de escritores franceses en esta moderna guía turístico-literaria de la mayor de las Baleares: quienes son citados, qué párrafos de qué obras se han seleccionado, el mayor o menor grado de pertinencia de los mismos, todo ello teniendo en cuenta que los textos viatorios constituyen una de las mejores expresiones del encuentro con el otro, es decir, de la alteridad y la interculturalidad. Intentaremos ver, asimismo, si a partir de las diferentes citas se establece un verdadero diálogo intercultural o si, por el contrario, la Guía-aplicación va dirigida, no al visitante foráneo, sino al autóctono; no al turismo real del que vive la isla, sino a un turismo identitario. ¿Nos movemos en el terreno de lo cultural, de lo intercultural? ¿en una zona fronteriza?

1 Le Monde, 19 janvier 1979. Ver cyrano.blog.lemonde.fr. [27/04/2021]. 


\section{En torno a la aplicación: algunas consideraciones}

Con la pretensión de dar a conocer el patrimonio literario de la isla, en 2016 el Consell Insular de Mallorca lanzó la aplicación² de autoguía "WoW Mallorca Literaria”, que forma parte de un proyecto más amplio y, de acuerdo con la página web, ofrece:

Una insólita ventana para [...] completar la experiencia de visita a cada sitio con una visión patrimonial y cultural más amplia. Entre sus prestaciones destaca la realidad aumentada al servicio del descubrimiento del entorno, el uso de tecnología iBeacon para facilitar la navegación al margen de la red wifi y la descarga vinculada a la proximidad que potencia la realización de las rutas. El app incluye una videocreación para cada sitio, con la lectura de la cita literaria a cargo de personas vinculadas con los sitios, los autores y/o los textos. Cada vídeo ha sido concebido como una pieza de creación artística que incorpora en lenguaje audiovisual nuevas claves interpretativas al usuario para conectar con los valores del sitio y del texto ${ }^{3}$.

De esta definición retenemos tres sintagmas. El usuario está ante una insólita ventana, imagen acertada puesto que la aplicación ofrece fácil acceso a visiones de la isla que la alejan de los estereotipos y caminos trillados. El imaginario de la ventana abre sobre paisajes en tres dimensiones, a lo que contribuye la realidad aumentada que "permite añadir capas de información visual sobre el mundo real que nos rodea [...]. Esto nos ayuda a generar experiencias que aportan un conocimiento relevante sobre nuestro entorno", lo que procura una mejor comprensión del mismo gracias a nuevas claves interpretativas que llegan tanto en formato escrito como audiovisual.

En palabras de la directora de la Fundación Mallorca Literaria, Carme Castells, la aplicación es "una invitación a recorrer Mallorca" siguiendo los pasos de los artistas que han escrito sobre ella. Las palabras de Jules Verne, Frédéric Chopin, George Sand, Robert Graves, Agatha Christie, Julio Cortázar o Josep Pla "trazan nuevos caminos en el paisaje y otorgan matices a nuestra visión de cada lugar visitado 5 ". La escritura se revela, pues, como re-creadora de territorios / paisajes, tanto urbanos como rurales. Pero parece tratarse también de visibilizar el patrimonio literario regional, puesto que abundan autores locales y breves textos anónimos salidos de la tradición oral, el Cançoner ${ }^{6}$. Y es que el proyecto entiende por patrimonio literario

2 Con fecha 26/04/2021 se han contabilizado un total de 1.275 descargas para dispositivos Apple y 1.670 a través de Google Play, perteneciendo los usuarios básicamente a cuatro países: España, Francia, Alemania y Reino Unido. Fuente: Fundación Mallorca Literaria.

3 Ver http://walkingonwords.com/es/guia/ [12/04/2021].

4 Ver www.neosentec.com [12/04/2021].

5 Ver nota 3.

6 En este sentido, hay que tener en cuenta que la Fundación es miembro de la Asociación "Espais escrits. Xarxa del Patrimoni Literari Català", en cuyo mapa literario se puede consultar textos -más de dos millones- de la literatura catalana asociados a lugares geográficos repartidos por todo el mundo. Para más información consultar la página web: www.espaisescrits.cat/que-hacemos/mapa-literario-catalan. 
toda aquella literatura que contribuye a la configuración del paisaje de la isla. Implica tanto las obras creadas por autores que han nacido y vivido en Mallorca, como las literaturas creadas por autores de otros lugares que han establecido un diálogo con la isla a través de cultura, historia, paisaje y patrimonio. (Castells $\left.{ }^{7}\right)$

Sea como sea, la aplicación responde a un esfuerzo por repensar y recalificar el territorio balear mediante dispositivos digitales que modernizan los documentos puestos a disposición del turista. Modernización tanto más necesaria cuanto que se trata de textos literarios, alejados a priori del turismo de sol y playa habitual en el archipiélago. El proyecto responde bien a la Carta del ICOMOS sobre turismo cultural, fechada en 1999, cuyo primer principio es considerar el turismo como un vehículo excepcional para el intercambio cultural y entender que

su conservación debería proporcionar oportunidades responsables y bien gestionadas a los integrantes de la comunidad anfitriona, así como proporcionar a los visitantes la experimentación y comprensión inmediata de la cultura y patrimonio de esta comunidad. (ICOMOS, 1999: 3)

Debemos notar que en 1999 la palabra patrimonio tenía ya un sentido amplio y, lejos de ser casi sinónimo de monumento o de museo, el concepto englobaba, como también hoy, todo aquello que forma parte del legado cultural de un pueblo: floklore, tradiciones, artesanía $\mathrm{y}$, no podía ser de otro modo, la literatura, que establece puentes entre la memoria colectiva de la comunidad anfitriona, el patrimonio material o inmaterial ${ }^{8}$, y el visitante. Este último se identifica con el turista literario, interesado bien en seguir los pasos de un autor o personaje

7 A partir de ahora, para la información proporcionada por Carme Castells, vía correo electrónico, señalaremos (Castells) al final de la cita. Vaya por delante nuestro agradecimiento, tanto a ella como a Joana Abrines, por la disposición y ayuda prestada.

8 Convención para la salvaguardia del patrimonio cultural inmaterial 2003. París, 17 de octubre de 2003.

I. Disposiciones generales - Artículo 2: Definiciones.

A los efectos de la presente Convención, 1. Se entiende por "patrimonio cultural inmaterial" los usos, representaciones, expresiones, conocimientos y técnicas -junto con los instrumentos, objetos, artefactos y espacios culturales que les son inherentes- que las comunidades, los grupos y en algunos casos los individuos reconozcan como parte integrante de su patrimonio cultural. Este patrimonio cultural inmaterial, que se transmite de generación en generación, es recreado constantemente por las comunidades y grupos en función de su entorno, su interacción con la naturaleza y su historia, infundiéndoles un sentimiento de identidad y continuidad y contribuyendo así a promover el respeto de la diversidad cultural y la creatividad humana. A los efectos de la presente Convención, se tendrá en cuenta únicamente el patrimonio cultural inmaterial que sea compatible con los instrumentos internacionales de derechos humanos existentes y con los imperativos de respeto mutuo entre comunidades, grupos e individuos y de desarrollo sostenible. 2. El "patrimonio cultural inmaterial", según se define en el párrafo 1 supra, se manifiesta en particular en los ámbitos siguientes: a) tradiciones y expresiones orales, incluido el idioma como vehículo del patrimonio cultural inmaterial; b) artes del espectáculo; c) usos sociales, rituales y actos festivos; d) conocimientos y usos relacionados con la naturaleza y el universo; e) técnicas artesanales tradicionales.

http://portal.unesco.org/es/ev.php-URL_ID=17716\&URL_DO=DO_TOPIC\&URL_SECTION=201.html [24/04/2021]. 
por un determinado territorio, bien en conocer los paisajes de un novelista o poeta o bien, siguiendo un recorrido inverso, en entender la re-creación que la escritura hace de determinados lugares quizás ya conocidos por el visitante ${ }^{9}$. En estos casos, la lógica quiere que la lectura haya precedido al viaje (o la visita), incluso cuando el itinerario es promovido por diferentes instituciones (Ayuntamientos, Diputaciones etc.) u Oficinas de Turismo, en lo que no deja de ser una operación de marketing. Entendemos que quien visita el París de Cortázar es un buen conocedor de la obra del escritor argentino, lo mismo que quien recorre el Madrid de Galdós, el Dublin de Joyce o La Mancha de Cervantes. De lo contrario se perderían los matices con los que estas obras revalorizan los lugares y se vería comprometido el diálogo intercultural.

No parece ser así en el caso de "WoW Mallorca Literaria"10. Entendiendo su viaje como de experiencia, el turista / visitante va descubriendo a lo largo de su deambular los textos y escritores que un día tuvieron relación con la isla: no la obra de un escritor, ni siquiera uno de sus títulos, sino tan sólo breves líneas de una novela, de un ensayo, de un relato de viajes, que no es necesario conocer en su totalidad. De fragmento en fragmento, a través del dispositivo digital, uno se apropia del territorio re-construido como espacio literario: no interesan datos históricos, fechas, descripciones detalladas sino, insistimos, el territorio como constructo literario, el territorio reinventado por la palabra poética, es decir, el paisaje, definido como "la interpretación de lo que se ve en el país (territorio) cuando éste se contempla con mirada estética" (Maderuelo, 2005: 6).

\section{Rutas literarias y escritores franceses}

La aplicación permite geolocalizar en el mapa diferentes elementos pertenecientes al patrimonio material o inmaterial, incidiendo este último en "la palabra literaria y el patrimonio de leyendas". Una vez localizado en el mapa, el turista podrá acercarse al punto de interés, señalado con una baliza en la que se ha estampado un código QR que, una vez escaneado con el dispositivo móvil, le dará acceso a la información concreta de un escritor (Figura 1). El usuario puede igualmente, a partir del escaneo, descargarse la aplicación completa y, una vez descargada, seleccionar idioma de consulta (catalán, castellano, inglés o alemán) y bloque temático. De las cuatro posibilidades ${ }^{11}$, retenemos para este estudio el denominado "Rutas literarias".

9 En torno a los conceptos de turismo y turista literario existe una amplia literatura crítica. Ver, entre otros títulos y autores: Magadán, Marta \& Jesús Rivas. 2011. Turismo literario. Oviedo, Septem Ediciones. Magadán, Marta \& Jesús Rivas. 2011b. El libro como atractor turístico. Oviedo, Septem Ediciones. Vázquez, Ilda \& Noelia Araújo. 2018. Revisión del turismo literario y su estado en la actualidad Cuadernos Geográficos 57(1), 305-329.

10 Como tampoco de "Espais escrits. Xarxa del patrimoni literari català" o del más amplio "GéoCulture. Le Limousin vu par les artistes", que engloba no sólo escritores, sino también artistas plásticos, artesanos, cineastas, músicos...

11 "Mapa", "Rutas literarias", “Caminatas"y "Leyendas". Utiliza esta última la técnica denominada gamificación. 

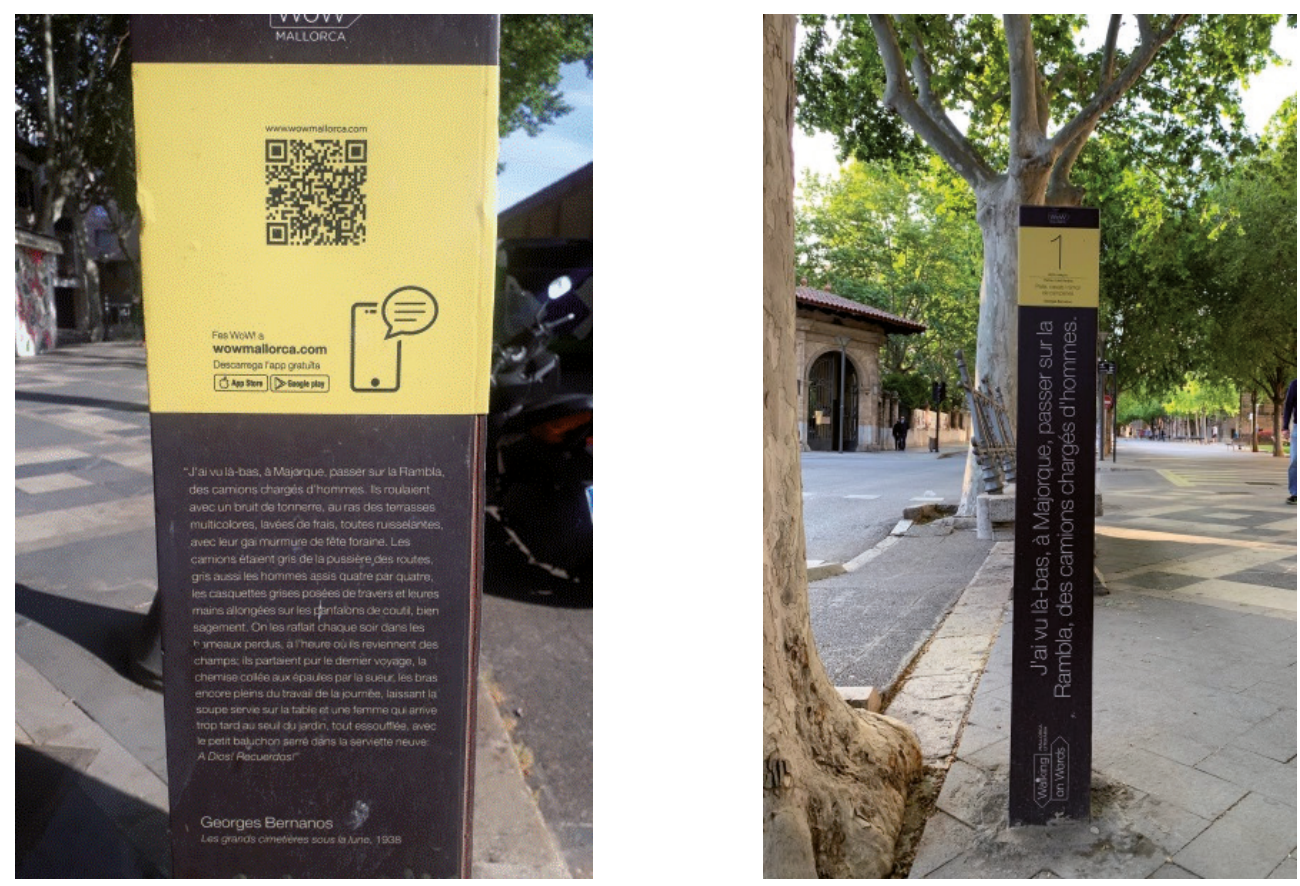

Figuras 1 y 2: Baliza situada al inicio de Las Ramblas de Palma, que contiene la cita de George Bernanos. Es visible el código QR desde el que descargarse bien la cita, bien la aplicación entera.

Se ofrecen siete rutas (Figura 3) correspondientes a siete áreas geográficas bien delimitadas. Al seleccionar una, el turista tiene acceso a una breve introducción de la misma y a un listado de escritores relacionados con el lugar (Figura 4-Ruta 4: Paisajes literarios de Tramuntana); 


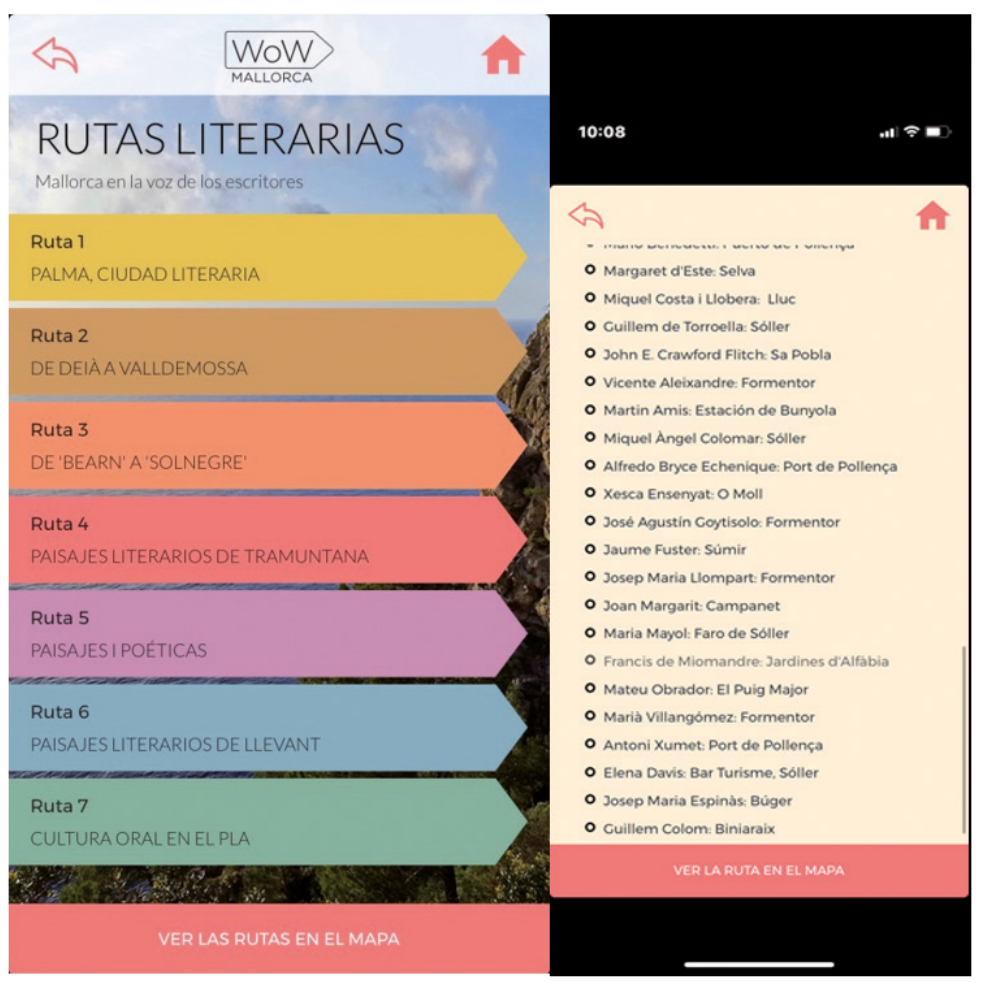

Figuras 3 y 4. Pantallazos en los que pueden apreciarse las siete rutas posibles y el despliegue (no completo) de escritores al pinchar, en este caso, sobre la Ruta 4 - Paisajes literarios de Tramuntana. Obsérvese que hay un único escritor francés, Francis de Miomandre.

Seguidamente tendrá que pinchar sobre uno de los nombres para acceder a:

- la cita literaria en el idioma seleccionado (Figuras 5 y 6 )

- una recreación en vídeo en la que se lee la cita en idioma original, con subtítulos al catalán (Figura 7)

- una breve nota explicativa de la relación del escritor con la isla en el idioma seleccionado, con posibilidad de escuchar el texto mediante enlace a un pod-cast.

- un histórico del lugar que, como en el caso anterior, puede ser leído o escuchado mediante enlace a un pod-cast. 


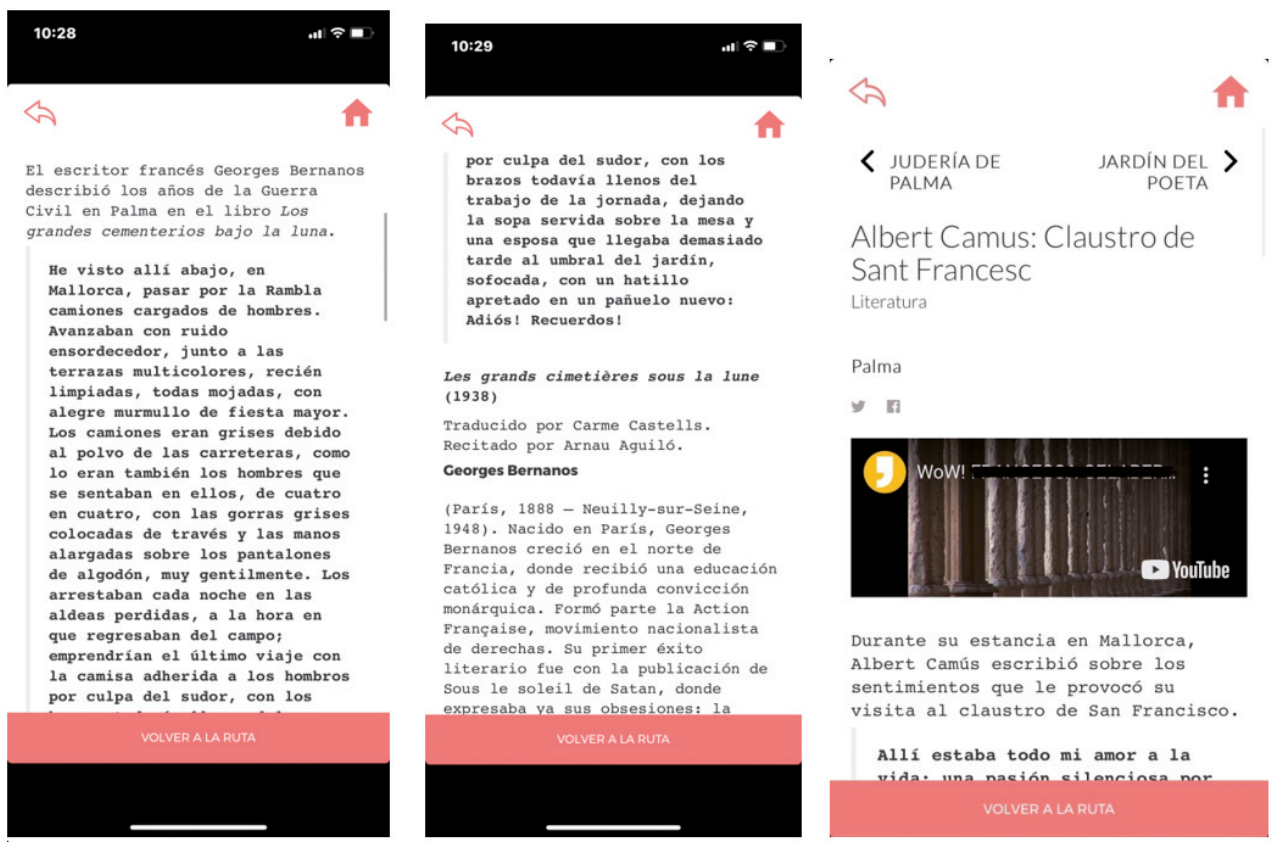

Figuras 5, 6 y 7. Pantallazos en los que puede verse la cita en la pantalla del móvil, una breve explicación sobre el autor y su relación con la isla, en este caso G. Bernanos. La última figura muestra la imagen desde la que puede accederse al video en el que se escenografía la cita literaria, en este caso la de A. Camus. (Se ha tachado el nombre de la persona que realiza la lectura dramática).

Se observa, pues, una jerarquía en la información, ligada a diferentes "grados de profundidad" en los que la capa más externa sería la baliza que, además de indicar el lugar en el que hay que detenerse, es portadora en si misma de información, y ello de manera doble: por las inscripciones que hay en ella y por el código QR, esta "ventana abierta" que vincula la baliza a la autoguía WoW Mallorca Literaria en formato app/web. Esto lleva a una segunda capa en la que el usuario tiene acceso a la diversidad de los datos que acabamos de exponer, en su versión de lectura, para acceder finalmente al video y a los audios. Todo el proceso gira así en torno a la cita literaria, que se convierte en un enunciado repetido hasta cuatro veces: en la baliza, en el texto escrito en la pantalla del móvil o la tableta, y en el vídeo, donde además de leída, la cita aparece de nuevo escrita en forma de subtítulos en catalán. En este contexto, la baliza es un objeto con una marcada carga "intencional", tomando la terminología de Paveau y Rosier, quienes definen el concepto de affordance como "une propriété d'un objet ou un trait de l'environnement immédiat, qui indique quelle relation l'agent humain doit instaurer avec l'objet (comment on doit s'en servir, ce qu'on doit faire avec)" (Paveau \& Rosier, 2010:185). En esta línea, la baliza, y en ella el código QR, proponen unas affordances 
discursives en tanto que "instruisent, prescrivent ou contraignent certaines activités langagières ou discursives" (Paveau \& Rosier, 2010: 186).

Heredera de la Guía turística en papel, esta guía digital temática -que, al contrario de otras guías, no permite interacción alguna- señala, como aquella, altos en el camino, lugares en los que vale la pena detenerse en detrimento de otros. Pero se cuestiona al tiempo la manera clásica de visitar un lugar y se establece una nueva relación entre el hombre y el territorio y/o paisaje, que se aprehende bajo el prisma de la literatura. Se establece también, sin duda, una nueva relación del hombre con el texto literario. La aplicación cuenta a día de hoy con un total de 149 citas, de las tan sólo 8 (un 5’3\%) corresponden a escritores en lengua francesa, repartidas del siguiente modo:

o Ruta 1 - Palma, ciudad literaria: Georges Bernanos, Albert Camus y Jean Giono (3 de 33).

o Ruta 2 - De Deià a Valldemossa: Jules Verne y George Sand (2 de 21).

o Ruta 3 - De Bearn a Solnegre: Gaston Vuillier (1 de 17).

o Ruta 4 - Paisajes Literarios de Tramuntana: Francis de Miomandre (1 de 33).

o Ruta 6 - Paisajes Literarios de Llevant: Paul Morand (1 de 16).

Observemos que no figura ningún escritor francés ni en la ruta 5 ni en la 7 y que si la ruta 1 puede recorrerse a pie, para los demás trayectos es necesario un vehículo; la primera ruta es (básicamente) urbana, el resto valoriza el paisaje entendiendo que "sólo hay paisaje cuando hay interpretación y esta es siempre subjetiva, reservada y poética o, si se quiere, estética" (Maderuelo, 2005: 35). En cuanto a las citas de los escritores franceses (importante es incidir en que han sido seleccionadas), sólo tres podían entenderse como descripciones en el sentido que Pierre Larousse dio al término y conviene a la definición de paisaje antes señalada:

La description littéraire [...] est la nature vue par un esprit particulier sous un jour propre à ses idées et à ses sentiments, la nature reproduite avec exactitude dans ses lignes principales, mais modifiée dans ses détails selon l'âme du poète et le sentiment qui le domine au moment où il la voit. (Larousse, 1870: 541)

Las citas en cuestión son las de Jean Giono, relativa al paisaje que rodea Son Sardina, núcleo poblacional a 5'5 kilómetros de Palma capital; Paul Morand para las Cuevas del $\operatorname{Drach}^{12}$ y Francis de Miomandre refiriéndose a los Jardines de Alfabia, todas ellas reproducidas en el anexo. Como se aprecia, ni una ni otra pueden considerarse fotografías del paisaje, sino que están dominadas por lo subjetivo y lo poético. Siguiendo a Maderuelo y a Ansón, podemos decir que todas ellas revelan el paisaje, no el territorio. Invitan a una ensoñación de

12 Se trata seguramente de un error, pues Morand se refiere en realidad a las cuevas de Artá. 
Anales de Filología Francesa, n. ${ }^{\circ}$ 29, 2021

LITERATURA FRANCESA DE VIAJES Y CÓDIGOS QR. ESPACIOS CULTURALES, ESPACIOS...

olores, formas y colores que tibiamente envuelven al lector. Frente al turista que realiza un viaje territorial, cuya misión es

levantar acta de la realidad visual de los territorios delimitados por la herramienta simbólica del mapa [...], la finalidad del viajero no es llegar a destino, sino el viaje propiamente dicho [...]. El lugar del turista, del viajero territorial, está enajenado. Unos y otros están ahí para nombrar, para poner nombres. El lugar del paisaje es el paisaje mismo, la experiencia de viajar [...]. Paisaje y viajero son la misma cosa." (Ansón, 2008: 239)

Esta reflexión bien podría ser la de Giono, que sirve como epígrafe del libro y figura también al inicio de la página web: "Vous voulez faire un voyage ? C'est maintenant que vous le faites, sur vos deux pieds, avec vos cinq sens". La experiencia intercultural del viajero amplía su horizonte con las palabras de P. Morand, que despliega la imaginación del lector al remitir al universo pictórico de Brueghel, Piranesi, W. Blake o G. Doré y las de F. de Miomandre, que evoca un oriente idílico, cayendo en el tópico del orientalismo insular.

Las palabras seleccionadas de George Sand, muestran un paisaje romántico, con utilización de epítetos o de adjetivos demasiado manidos ("ciel magnifique", "île enchantée", "l'air si pur", "la mer si bleue") que poco aportan a la descripción del lugar. En este caso parece interesar más ofrecer datos autobiográficos referidos a su estancia en Mallorca: por un lado, la cita pertenece a Histoire de ma vie, y no al más esperado Un hiver à Majorque; por otro Sand sólo da noticia de sus acompañantes en la aventura mallorquina: "mes enfants", con quienes ejercía de maestra, y "le malade", entiéndase Chopin, al que acusa indirectamente del fracaso de la estancia en la isla.

"S'il est une contrée que l'on puisse connaître à fond sans l'avoir jamais visitée, c'est ce magnifique archipel des Baléares". Con estas palabras se abre la cita seleccionada de Jules Verne, como también el capítulo VI de Clovis Dardentor. Basta, continúa el narrador, con encerrarse

dans une bibliothèque, à la condition que cette bibliothèque possédât l'ouvrage de Son Altesse l'archiduc Louis-Salvator d'Autriche sur les Baléares, d'en lire le texte si complet et si précis, d'en regarder les gravures en couleurs, les vues, les dessins, les croquis, les plans, les cartes, qui font de cette publication une œuvre sans rivale. C'est, en effet, un travail incomparable pour la beauté de l'exécution, pour sa valeur géographique, ethnique, statistique, artistique... (Verne, 1896: 103)

El personaje homónimo de la novela recorre Palma desde el muelle, donde desembarcan los pasajeros del Argelès, hasta el castillo de Bellver. No deja de ser curioso, pues, que se desplace a Verne de la ruta 1 a la 2 y ello no para detenerse en la descripción de los parajes por los que transcurre dicha ruta, sino para referirse a una persona emblemática, el Archiduque Luis Salvador de Austria, que compró grandes extensiones de tierra en la costa 
entre Deià y Valldemossa, fijó su residencia mallorquina en La Estaca y escribió una obra magna sobre el archipiélago: Las Baleares descritas por la palabra y el dibujo (1869). Es decir, nada sabemos de la Palma de Jules Verne ${ }^{13}$, sino que la cita funciona como una llamada a la competencia lectora del turista/visitante. Al remitir a las descripciones de la obra del Archiduque, se establece un diálogo a tres por medio de la intertextualidad, solicitando siempre la connivencia del turista/lector. El diálogo intertextual no acaba aquí, pues en otra cita de esta misma ruta, Julio Cortázar describe una puesta de sol contemplada desde las tierras del Archiduque, con estas palabras:

Del sol quedaba un último, frágil segmento anaranjado. Lo vimos desaparecer detrás del perfecto borde del mar, envuelto en el halo que aún duraría algunos minutos. Y entonces surgió el rayo verde, no era un rayo sino un fulgor, una chispa instantánea en un punto como de fusión alquímica, de solución heracliteana de elementos. Era una chispa intensamente verde, era un rayo verde, aunque no fuera un rayo, era el rayo verde, era Julio Verne murmurándome al oído: “¿Lo viste al fin, gran tonto? ${ }^{14 ”}$

"El rayo verde" de Cortázar nos lleva a la novela de igual título de Verne quien, a su vez, conduce al Archiduque, siguiendo un complejo entramado intertextual / intercultural que, insistimos, para ser enteramente descifrado requiere de los conocimientos previos del turista. Algo parecido sucede con las palabras de Gaston Vuillier en lo que se espera sea una descripción del Jardín de Raixa. Lejos de esto, al desencriptar el mensaje salen a la luz dos nombres: el del Cardenal Despuig ${ }^{15}$, fundador del museo de Raixa, y el de George Sand, con la famosa anécdota del tintero que... ¿conoce el visitante no familiarizado con la literatura francesa? ¿tiene la suficiente transcendencia como para ser destacada?

Volviendo a Palma, debemos hacernos eco de las palabras de Georges Bernanos y de Albert Camus sobre la capital de la isla. El primero, a pesar de residir en Mallorca varios meses, se refirió poco a ella, absorto como estaba en la redacción de Journal d'un curé de campagne, luego, a partir de enero de 1937, de Grands cimetières sous la lune, donde, aun haciendo de manera genérica hermosas alusiones a la isla, expone sus vivencias del estallido de la guerra Civil tal como lo vivió en Mallorca. De ahí que se haya seleccionado una referen-

13 Véase, a modo de ejemplo, esta descripción de Palma vista desde el Castillo de Bellver: "Au pied du castillo, s'abaisse la colline, revêtue de son noir manteau de pins d'Alep. Au -delà se groupe le charmant faubourg de Terreno. Plus bas, s'arrondit la baie toute bleue, tachetée de petits points blancs qu'on eût crus des oiseaux de mer et qui ne sont que des voiles de tartanes. Plus loin, se développe la ville en amphithéâtre, sa cathédrale, ses palais, ses églises, ensemble éclatant, baigné dans cette atmosphère lumineuse que le soleil crible de rayons dorés, lorsqu'il décline vers l'horizon. Enfin, au large, resplendit la mer immense, avec çà et là des navires déployant leur blanche voilure, des steamers balayant le ciel de leur longue queue fuligineuse" (Verne, 1896: 128).

14 De "El rayo verde", en Papeles inesperados, Alfaguara, Madrid, 2009. La cita está directamente cogida de la aplicación.

15 Antonio Despuig y Dameto (Palma de Mallorca, 1745-Lucca, 1813). Señalemos que, según cuenta George Sand en Un hiver à Majorque, la tinta se derrama sobre un mapa náutico que, adquirido por el Cardenal Despuig, habría pertenecido a Américo Vespucio. 
cia a los camiones cargados de hombres que iban a ser fusilados a su paso por Las Ramblas. Un solo elemento paratextual, la fecha, remite al conflicto armado. Un año antes del mismo, Albert Camus había pisado tierra mallorquina y dejado constancia de ello en el breve ensayo "Amour de vivre". En este caso, a las hermosas líneas dedicadas al barrio antiguo de Palma, al silencio de sus callejas estrechas, a la inmensa paz que invadió al narrador durante su visita al Claustro de la Basílica de San Francisco:

Et marchant le long des maisons, m'arrêtant dans les cours pleins de plantes vertes et de piliers ronds et gris, je me fondais dans cette odeur de silence [...]. Je passais aussi de longues heures dans le petit cloître gothique de San Francisco. Sa fine et précieuse colonnade luisait de ce beau jaune doré qu'ont les vieux monuments en Espagne. Dans la cour, des lauriers-roses, de faux poivriers, un puits de fer forgé d'où pendait une longue cuillier de métal rouillé. (Camus, 1965: 43)

a estas líneas, insisto, se prefieren las palabras, igualmente hermosas, que dan testimonio de este humanismo mediterráneo característico del pensamiento camusiano, pero son poco descriptivas del lugar (ver Anexo). También en este caso, el abanico cultural se ensancha con la llamada a los Apolos dóricos y a la pintura de Giotto.

\section{De lo cultural a lo inter-cultural}

WoW Mallorca Literaria "reivindica la literatura como un medio cultural de transmisión de conocimientos de primer orden a nivel artístico, para dar a conocer Mallorca y las voces de sus paisajes" (Castells). Voces creadoras de paisajes, escritores autóctonos, españoles y extranjeros, leyendas... Como se ha visto -a pesar de la menguada presencia de escritores franceses- el despliegue de referencias culturales es ambicioso, así como el conjunto de escritores seleccionados para cada ruta. En la ruta 4 a la que ya nos hemos referido se codean Agatha Christie y Santiago Rusiñol, Berenguer d'Anoia y Mario Benedetti, Margaret d'Este y Brice Echenique, Josep Pla y Camilo José Cela. Por detenernos en un caso excepcional, la cita seleccionada de Berenguer d'Anoia, en occitano, pertenece a Mirall de trobar (ca. 1300-1340) obra, leemos, "sobre los preceptos, la poética, doctrina gramatical y retórica" medievales, en absoluto relacionada con la esperada descripción del casco histórico de Inca. Así, más allá de la re-creación de un paisaje por medio de la palabra poética, se re-convierte el territorio insular en espacio literario a todos los efectos, se revaloriza.

Es cierto que las diferentes literaturas representadas pertenecen a sistemas literarios y códigos sociales muy similares, lo que lleva a preguntarse por el diálogo intercultural que puede establecerse entre los actores en juego, tanto más cuanto que la conciencia de la alteridad se sitúa en el centro de los estudios interculturales. Es evidente que no podemos abordar un acercamiento a lo intercultural del modo en que lo harían, por ejemplo, los estudios co- 
loniales o postcoloniales o el análisis de los estereotipos en la literatura de viaje, definida en parte por su encuentro con la cultura del otro.

De las citas de escritores franceses únicamente una, la de Francis de Miomandre, traslada una imagen estereotipada de la isla, al remitirnos al orientalismo tanto del lugar como de los habitantes; a un ailleurs que, ligado a la necesidad de evasión, pusieron de moda los viajeros románticos como Joseph Bonaventure Laurens quien, al igual que Miomandre, destacaba el aspecto mil y una noches de los jardines de Alfabia ${ }^{16}$. Una segunda cita, la de G. Bernanos, tiene un marcado carácter político sobre un tema que dividió a la sociedad española y en la se implicaron conocidos políticos e intelectuales franceses. Y sin embargo, ante un receptor que no tenga las competencias requeridas tanto en los acontecimientos históricos a los que se hace referencia como en la compleja figura y obra de Bernanos, la cita queda marcada por la opacidad, se pierden los matices y se pone en riesgo la mejor comprensión "de" y "entre" los pueblos. Por lo demás, y con buen criterio, se han dejado de lado relatos (o fragmentos) con una alta carga de prejuicios y clichés de todo tipo, que bien podrían invitar a un estudio imagológico, como los presentes en Un hiver à Majorque, de George Sand. Todo ello quedaba fuera, evidentemente, del propósito del proyecto.

Es sobre todo la Ruta 7 - Cultura oral en el Pla, la que pondrá al turista en contacto con el otro, con lo que podría denominarse "mallorquinidad". Subtitulada "Mallorca con voz propia", se abre con las siguientes palabras de Rafel Ginard" : "Para conocer la auténtica historia del alma colectiva de Mallorca, no hurguéis en los archivos llenos de polvo y polillas, sino revolcaos, sumergíos en el folklore”. En esta ruta comenzaría el verdadero diálogo intercultural, a condición de que visitara la zona, donde la afluencia de turistas es pobre, el turista extranjero. El espacio existente "entre" o "en medio de" dos culturas, marcado por el prefijo inter-, ocuparía en este caso un lugar central. Sin embargo, las excursiones guiadas dirigidas, en gran parte, a centros escolares, parecen destinar la ruta a un turismo identitario.

Aun así, una mirada panorámica a la aplicación, como si de un paisaje se tratara, permite entenderla en su complejidad. Sólo si imaginamos que cada cita es una simple, aunque hermosa, tesela, comprenderemos el extraordinario valor literario y cultural del mosaico que surge de la unión de todas ellas. Y es que, como señala el semiótico de la cultura, Iuri Lotman:

16 Veamos un ejemplo: “Ce n'est que dans les jardins d'Alfabia [...] que j'ai pu me faire illusion de me croire dans une autre Alhambra. Là, des eaux jaillissantes, des plates-bandes garnies des fleurs recherchées, des bosquets de citronniers et d'orangers chargés de fruits, des bosquets de palmiers, des berceaux de jasmin à grandes fleurs, représentaient assez bien un de ces délicieux séjours [...]" (Laurens, 1945: 127).

17 Rafel Ginard Bauçà (1899-1976) sacerdote, folklorista y poeta mallorquín. Entre 1966 y 1974 recopiló en el Cançoner popular de Mallorca más de 15.000 canciones populares. Fue también un estudioso de la obra de Ramón Llull, encargándose de los prólogos del Llibre d'Evast e Blanquerna (1948) y del volumen XXI de les Obres de Ramon Llull (1950). 
La cultura en su totalidad puede ser considerada como un texto. Pero es extraordinariamente importante subrayar que es un texto complejamente organizado, que se descompone en una jerarquía de "textos en el texto" y que forma complejas entretejeduras de textos. (1996: 109)

Según Lotman, la cultura está hecha a base de textos de todo tipo (es decir, en diferentes lenguajes), cada cual con sus referentes, que a su vez se incorporan a una trama profundamente dinámica para convertirse en significado de un segundo texto. Los textos se convierten, pues, en espacios semióticos que interactúan entre ellos, establecen un diálogo y generan nuevos significados.

\section{Conclusión}

Novedosa guía turístico-literaria descargable a partir de un código QR, la aplicación WoW Mallorca Literaria invita al turista a conocer la isla siguiendo los pasos y la voz de diversos escritores extranjeros y autóctonos. La aplicación ofrece siete rutas correspondientes a siete áreas (o subáreas) geográficas cuyo hilo conductor es la palabra poética. Contemplado con mirada estética, a través de un mensaje semióticamente du/triplicado, el territorio se convierte en paisaje.

Un 5,3\% de las citas seleccionadas corresponden a autores en lengua francesa y han sido extraídas de libros que pueden englobarse, excepto la de Bernanos, en el género de literatura de viaje, lo que conlleva referirse a la alteridad y a la interculturalidad. Del estudio de estas citas se observa que el entramado intertextual despliega un amplio abanico de saberes que trascienden dichas citas, haciendo continuas llamadas a la competencia cultural del turista o visitante: a falta de los conocimientos requeridos, el diálogo intercultural quedaría envuelto en una cierta opacidad, aunque bien puede despertar la curiosidad del lector. Por otro lado, hay que referirse con cierta cautela al concepto de experiencia intercultural, dado que el lector de las citas no se abre a una cultura (en el sentido etnográfico de la palabra) muy diferente de la suya, ni establece un diálogo con ella que le permita interpelar la suya propia. En todo caso el espacio insular se convierte en un espacio en el que tienen lugar les relations d'échange; aisladas en las balizas de todo contexto (hablábamos en la Introducción de un fuera de campo discursivo), las diferentes citas establecen entre ellas una relación dialógica, siendo así capaces de generar nuevos significados, hecho que se convierte en uno de los grandes logros de la aplicación. 
Anales de Filología Francesa, n. ${ }^{\circ}$ 29, 2021

CARLOTA VICENS-PUJOL

\section{Referencias bibliográficas $^{18}$}

Ansón, Antonio. 2008. “Territorios y paisajes. Modelos para pensar fotografía y literatura, tal vez soñar" in Maderuelo, Javier (ed.). Paisaje y territorio. Huesca/Madrid, Adaba editores, 227-254.

Camus, Albert. 1965 [1937]. Essais. Paris, Gallimard.

Icomos. 1999. "Carta Internacional sobre Turismo Cultural": <https://www.icomos.org/ charters/tourism_sp.pdf> [11/04/2021].

LARousse, Pierre. 1870. Grand Dicctionnaire Universel du XIX bnf.fr/> [26/04/2021].

LAUREns, Joseph Bonaventure. 1945 [1840]. Souvenirs d'un voyage d'art à l'île de Majorque. Palma, Moll.

Lotman, Iuri. 1996. La semiosfera I. Semiótica de la cultura y del texto. Madrid, Frónesis, Cátedra.

Kundera, Milan. 1979. Le Monde, 19 enero.

Maderuelo, Javier. 2005. El paisaje. Génesis de un concepto. Madrid, Adaba editores.

NeOSEnTEC. 2020. "Realidad aumentada": <www.neosentec.com> [12/04/2021].

PAVEAU, Marie Anne \& Laurence ROSIER. 2010. "Le discours des objets. Pratiques et techniques de circulation, entre clandestinité et exhibition discursive" in Çedille. Revista de estudios franceses. Monografía 1, 178-196: <https://cedille.webs.ull.es/M1/paveau-rosier. pdf $>[8 / 04 / 2021]$.

Trapiello, Andrés. 2021. Madrid. Barcelona, Destino.

UNESCO. 2003. "Convención para la salvaguarda del patrimonio cultural inmaterial":

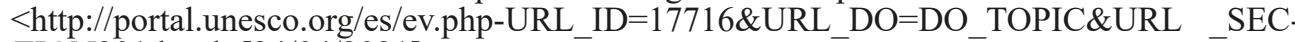
TION201.html $>[24 / 04 / 2021]$.

Verne, Jules. 1896. Clovis Dardentor. Paris, Hetzel.

WALKING ON WORDS. 2015. “APP de autoguía”: <http://walkingonwords.com/es/guia/> [22/04/2021]. Existe también edición en papel, Walkin on Words. Mallorca literaria. 7 rutas guiadas por el paraíso, si puedes resistirlo. Palma de Mallorca, Consell Insular.

18 Las citas de WoW Mallorca Literaria incluidas en este texto han sido directamente copiadas de la aplicación. Por tanto, se ofrecen únicamente las referencias bibliográficas de aquellas citas literarias aportadas por la autora del trabajo. 


\section{Anexo}

CAMUS, ALBERT - "Là était tout mon amour de vivre: une passion silencieuse pour ce qui allait peut-être m'échapper, une amertume sous une flamme. Chaque jour je quittais ce cloître comme enlevé à moi-même, inscrit pour un court instant dans la durée du monde. Et je sais bien pourquoi je pensais alors aux yeux sans regard des Apollons doriques ou aux personnages brûlants et figés de Giotto. C'est qu'à ce moment, je comprenais vraiment ce que pouvaient m'apporter de semblables pays. J'admire qu'on puisse trouver au bord de la Méditerranée des certitudes et des règles de vie, qu'on y satisfasse sa raison et qu'on y justifie un optimisme et un sens social. Car enfin, ce qui me frappait alors ce n'était pas un monde fait à la mesure de l'homme mais qui se refermait sur l'homme" (Albert Camus, Amour de vivre, L'envers et l'endroit, 1937) Ruta 1, Claustro de San Francisco.

BERNANOS, GEORGES - “J'ai vu sur là-bas, à Majorque passer, sur la Rambla des camions chargés d'hommes [...]. Les camions étaient gris de la poussière des routes, gris aussi les hommes assis quatre par quatre, les casquettes posées de travers et leurs mains allongées sur le pantalon de coutil, bien sagement. On les raflait chaque soir dans les hameaux perdus, à l'heures où ils reviennent des champs; ils partaient pour le dernier voyage, la chemise collée aux épaules par la sueur, les bras encore pleins du travail de la journée, laissant la soupe servie sur la table et une femme qui arrive trop tard au seuil du jardin, tout essoufflée, avec le petit baluchon serré dans la serviette neuve: A Dios! Recuerdos!" (Georges Bernanos, Les Grands cimetières sous la lune, 1938) Ruta 1, Las Ramblas.

GIONO, JEAN - “En dehors des grandes routes il n'y a que des chemins et des pistes, où il est possible de flâner en toute quiétude [...] Peu à peu on arrive à différencier le chant des oiseaux, le cri des insectes, le bruit velouté du vent dans les différents feuillages. Les odeurs -ne serait-ce que l'amertume des fleurs d'amandiers et celles de figuiers en sève- vous transportent plus rapidement que la plus moderne des caravelles. Vous voulez faire un voyage? C'est maintenant que vous le faites, sur vos deux pieds, avec vos cinq sens" (Jean Giono, "Les joies de l'île" en La chasse au bonheur, 1969) Ruta 1, Son Sardina.

MIOMANDRE, FRANCIS DE - "Les plus grands [jardins], comme ceux de Raixa ou d'Alfabia, ont ainsi un air de harems, cernés de murailles, invisibles de loin. On pourrait rôder autour d'eux longtemps, sans douter de leur présence, et, quand on y pénètre, cela semble toujours un peu par effraction.

Rien qui rende plus précieuse la vue d'un massif, d'un bassin, d'une treille, que ces précautions jalouses. On en demeure ému comme d'une révélation. Furtive, la favorite a soulevé un peu son voile, on a surpris le feu d'un regard, la rouge promesse d'un sourire" (Francis de Miomandre, Mallorca, 1933) Ruta 4, Jardines de Alfabia. 
MORAND, PAUL - “C'est l'inextricable, un vrai magasin de cordier; immenses salles en ficelée de pierre, colonie de coraux démesurés, bric-à-brac de marchand d'habits, jardin zoologique, franges de châle de Manille, saules pleureurs, chenillés et passementerie... Tout cela, sans l'aide des hommes; ni Breughel, ni le Piranèse des Prisons, ni William Blake, ni Gustave Doré, n'ont rien pu imaginer de tel. On étouffe dans le ventre de cette baleine qui est venue crever sur le rivage, entre les dents de ce monstre préhistorique, parmi ces larmes gelées qui nous surplombent, comme la glace sur le toit des chalets suisses" (Paul Morand, Majorque, 1963) Ruta 6, Coves del Drac - Manacor.

SAND, GEORGE - "Je quittai la Chartreuse avec un mélange de joie et de douleur. J'y aurais bien passé deux ou trois ans, seule avec mes enfants. Nous avions une malle de bons livres élémentaires que j'avais le temps de leur expliquer. Le ciel devenait magnifique et l'île un lieu enchanté [...]. De quelle poésie sa musique remplissait ce sanctuaire, même au milieu de ses plus douloureuses agitations! Et la Chartreuse était si belle sous ses festons de lierre, la floraison si splendide dans la vallée, l'air si pur sur notre montagne, la mer si bleue à l'horizon! C'est le plus bel endroit que j'aie jamais habité, et un des plus beaux que j'aie jamais vus. Et j'en avais à peine joui! N'osant quitter le malade, je ne pouvais sortir avec mes enfants qu'un instant chaque jour, et souvent pas du tout. J'étais très-malade moi-même de fatigue et de séquestration" (George Sand, Histoire de ma vie, 1855) Ruta 2, Miranda dels Lledoners - Valldemossa.

VERNE, JULES - “S'il est une contrée que l'on puisse connaître à fond sans l'avoir jamais visitée, c'est ce magnifique archipel des Baléares [...] Oui! si ce qui a été fait pour ces oasis de la mer méditerranéenne l'était pour n'importe quel autre pays des deux continents, il serait inutile de se déranger, de quitter sa maison, de se mettre en route, inutile d'aller de visu admirer les merveilles naturelles recommandées aux voyageurs. Il suffirait de s'enfermer dans une bibliothèque, à la condition que cette bibliothèque possédât l'ouvrage de Son Altesse l'Archiduc Louis-Salvator d'Autriche sur les Baléares, d'en lire le texte si complet et si précis, d'en regarder les gravures en couleurs, les vues, les dessins, les croquis, les plans, les cartes, qui font de cette publication une œuvre sans rivale. C'est, en effet, un travail incomparable pour la beauté de l'exécution, pour sa valeur géographique, ethnique, statistique, artistique..." (Jules Verne, Clovis Dardentor, 1896) Ruta 2, Son Marroig - Deià.

VUILLIER, GASTON - "Le cardinal Despuig ajouta au blason de sa famille la pourpre cardinalice, l'ordre de Charles III d'Espagne, dont il fut grand'croix, et le patriarcat d'Antioche. Il acheta en Italie au peintre écossais Hamilton, qui y pratiquait des fouilles, un temple que Domitien avait élevé à Égérie et il poursuivit les travaux à ses frais. De 1767 à 1768 il eut le bonheur de découvrir la plus grande partie des sculptures précieuses qui forment le musée actuel. Là je vis aussi la fameuse carte nautique sur laquelle George Sand est accusée d'avoir renversé l'encrier, mais encadrée, protégée par un verre et accrochée au mur" (Gaston Vuillier, Les îles oubliées. Les Baléares, la Corse et la Sardaigne. Impressions de voyage illustrées par l'auteur, 1893) Ruta 3, Jardín de Raixa. 
